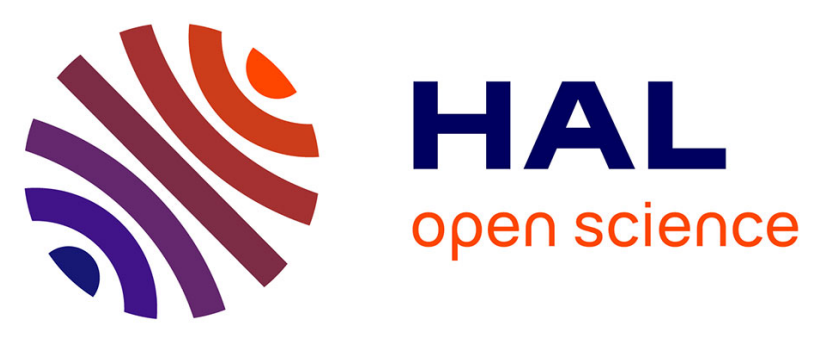

\title{
A Parametric Replay-Based Framework for Underwater Acoustic Communication Channel Simulation
}

François-Xavier Socheleau, Christophe Laot, Jean-Michel Passerieux

\section{To cite this version:}

François-Xavier Socheleau, Christophe Laot, Jean-Michel Passerieux. A Parametric ReplayBased Framework for Underwater Acoustic Communication Channel Simulation. UComms 2014: Underwater Communications and networking, Sep 2014, Sestri Levante, Italy. 10.1109/UComms.2014.7017148 . hal-01188803

\section{HAL Id: hal-01188803 https://hal.science/hal-01188803}

Submitted on 31 Aug 2015

HAL is a multi-disciplinary open access archive for the deposit and dissemination of scientific research documents, whether they are published or not. The documents may come from teaching and research institutions in France or abroad, or from public or private research centers.
L'archive ouverte pluridisciplinaire HAL, est destinée au dépôt et à la diffusion de documents scientifiques de niveau recherche, publiés ou non, émanant des établissements d'enseignement et de recherche français ou étrangers, des laboratoires publics ou privés. 


\title{
A Parametric Replay-Based Framework for Underwater Acoustic Communication Channel Simulation
}

\author{
Francois-Xavier Socheleau, Christophe Laot, Jean-Michel Passerieux
}

\begin{abstract}
This paper lays the foundation of an underwater acoustic channel simulation methodology that is halfway between parametric modeling and stochastic replay of at-sea measurements of channel impulse responses. The motivation behind this approach is to extend the scope of use of replay-based methods by allowing some parameterization of the channel properties while complying with some level of realism. Based on a relative entropy minimization between the original channel impulse response and the simulated one, the idea is to deliberately distort the original channel statistics in order to meet some specified constraints.
\end{abstract}

Index Terms-Underwater acoustic communications, channel modeling, stochastic replay, relative entropy minimization

\section{INTRODUCTION}

A MONG the various simulation strategies proposed in the literature, stochastic replay of time-varying impulse response (TVIR) measured in situ has emerged as a relevant and accurate underwater acoustic communication (UAC) channel simulation method. As described in [1]-[3], the idea is to probe a UAC channel at sea to then generate in laboratory new random TVIR with statistical properties similar to the original measurement. From a single measurement, it is thus possible to compare competing transmission schemes when faced to the same realistic environment [3]. Thanks to Monte-Carlo simulations, design and validation metrics such as bit error rate [1]-[3], capacity bounds [4]-[6] or fading statistics [2] can be computed with a good accuracy. Note however that the methodology behind stochastic replay does not apply to every UAC channel. Cyclostationary channels are typical examples that fall outside the domain of applicability of such a method (ref. to [3] for more details).

When designing channel models, there is a always a choice to make between realism, ease of parameterization (knowledge of the model parameters should be easy to acquire) and flexibility (the diversity of the simulated environments should be controlled to some extent). Standard stochastic replay meets the first two criteria but not the last one. Indeed, the main drawback of stochastic replay lies in its lack of diversity. As opposed to fully parametric UAC channel modeling, the input

F.-X. Socheleau is with ENSTA Bretagne, UMR CNRS 6285 Lab-STICC, Ueb, France (e-mail: francois-xavier.socheleau@ensta-bretagne.fr).

C. Laot is with the Institut Mines-Telecom, Telecom Bretagne, UMR CNRS 6285 Lab-STICC, Ueb, France (e-mail: christophe.laot@ telecom-bretagne.eu). J.-M. Passerieux is with Thales Underwater Systems, France (e-mail: jeanmichel.passerieux@fr.thalesgroup.com). impulse response that drives the replay-based simulator corresponds to a specific transmission configuration in a specific environment leading to fixed channel statistical properties. As shown in [1], [7], some degree of diversity can however be achieved by "building a database with channels probed in different areas, different seasons, etc.". The lack of flexibility of replay-based models can be a concern when extensive modem testing is called for in order to assess the robustness of communication links.

The purpose of this paper is to show that this drawback can be compensated, to a certain extent, by creating diversity in some statistical sense. Existing replay-based methods already artificially create some environment diversity by acting on the noise properties only. From a single TVIR, several acoustic environments can be simulated by changing the noise power or the noise distribution. Higher level of diversity can be achieved by exploiting the ability of common replay-based methods to separate first-order statistics of the measured impulse response from second-order statistics [1], [2]. A first example was given in [8] where the power ratios between the specular and the scattered components of the channel were artificially modified to test the receiver robustness to random scattering.

In this work, we extend this idea and seek to build a replay-based simulation strategy that allows some level of parameterization in order to enlarge its scope of use. The problem we want to tackle is the following: given prior information on UAC channels that is available through a measured impulse response, can we build a channel model that satisfies some specified constraints while being "as close as possible" to the original TVIR? Constraining the model means that we want to control some of its properties through specific parameters such as the Doppler spread or the level of taps correlation for instance. "Being as close as possible" is a way to achieve some level of realism as provided by the original TVIR. As shown in the sequel, this problem can be addressed by optimizing an information-theoretic criterion that formally defines the concept of model proximity. The results presented in the sequel are the first steps towards the design of this parametric replay-based framework. For now, we only focus on second-order statistics and only consider constraints on the channel Doppler spread and the channel energy. In addition, we mostly insist on the modeling strategy rather than on its applicability with real data.

The paper is organized as follows: Section II is devoted to the presentation of the replay-based channel simulation strategy which our framework is based on. Section III presents 
the method used to deliberately distort the original TVIR statistics in order to meet the desired constraints. Numerical examples are provided in Section IV. Finally, perspectives are discussed in Section V.

\section{REPLAY-BASED CHANNEL MODEL}

We consider a doubly selective underwater acoustic channel, modeled as a random linear time-varying system $\mathbb{H}$ that maps input signals $x(t)$ onto output signals $y(t)$ according to the I/O relationship

$$
y(t)=(\mathbb{H} x)(t)+w(t)=\int_{\tau} h_{\mathbb{H}}(\tau, t) x(t-\tau) d \tau+w(t),
$$

where $h_{\mathbb{H}}(\tau, t)$ is the channel impulse response and $w(t)$ denotes the ambient noise. As common practice in replaybased simulation, the input of the simulator is a discrete-time baseband estimate of the channel impulse response whose mean Doppler shift has been removed [1]-[3]. Such an estimate is denoted as $h_{l}(k)$ where $l \in\{0, \cdots, L-1\}$ is the tap index and $k \in\{0, \cdots, K-1\}$ is the time index. In agreement with [2], $\left\{h_{l}(k)\right\}$ is modeled as a multi-variate trend stationary random process so that, for all $k, k_{1}$ and $k_{2} \in \mathbb{Z}$

$$
h_{l}(k)=\bar{h}_{l}(k)+\tilde{h}_{l}(k) \text {, }
$$

with

$$
\mathbb{E}\left\{h_{l}(k)\right\}=\bar{h}_{l}(k),
$$

and

$$
\begin{aligned}
& \mathbb{E}\left\{\tilde{h}_{l}\left(k_{1}\right) \tilde{h}_{p}^{*}\left(k_{2}\right)\right\}=\mathbb{E}\left\{\tilde{h}_{l}(k) \tilde{h}_{p}^{*}\left(k+k_{2}-k_{1}\right)\right\}, \\
& \mathbb{E}\left\{\tilde{h}_{l}\left(k_{1}\right) \tilde{h}_{p}\left(k_{2}\right)\right\}=\mathbb{E}\left\{\tilde{h}_{l}(k) \tilde{h}_{p}\left(k+k_{2}-k_{1}\right)\right\} .
\end{aligned}
$$

$\bar{h}_{l}(k)$ is called the trend and is a slowly time-varying deterministic component. $\tilde{h}_{l}(k)$ is a zero-mean wide-sense stationary random process assumed to be Gaussian. This model describes the UA channel as a multivariate Rician fading process with a slowly time-varying mean. $\bar{h}_{l}(k)$ can be interpreted as the contribution of (pseudo) deterministic physical phenomena to channel fluctuations and $\tilde{h}_{l}(k)$ represents the channel fluctuations attributable to scatterers that result in fast fading. Note that since no particular assumption is made about the correlation of scatterers, the model is very general and includes the wide-sense stationary uncorrelated scattering (WSSUS) model as a subset.

Based on (2), standard replay methods first consist in isolating both components $\bar{h}_{l}(k)$ and $\tilde{h}_{l}(k)$ from $h_{l}(k)$. This can be done by estimating the specular component $\bar{h}_{l}(k)$ either by simple time averaging when it is time-invariant [1], [3], [9] or by an empirical mode decomposition [2] in the general timevarying case. $\tilde{h}_{l}(k)$ is then obtained as a difference between $h_{l}(k)$ and $\bar{h}_{l}(k)$. Finally, stochastic replay consists in drawing new realizations of the channel random components based on the observation $\tilde{h}_{l}(k)$. These realizations are then added to the original specular components $\bar{h}_{l}(k)$ to obtain a new TVIR. New realizations of $\left\{\tilde{h}_{l}(k)\right\}$ can be obtained either by explicitly estimating the second order statistics of $\left\{\tilde{h}_{l}(k)\right\}$, as given by the scattering function for instance [1], and by then filtering white Gaussian noises, or they can be generated by simply adding noise on the phase of the discrete Fourier transform of the observation $\tilde{h}_{l}(k)$ [2], [10].

In this work, we consider the first approach and assume that we are able to estimate the second order statistics of $\left\{\tilde{h}_{l}(k)\right\}$ given by the $2 L \times 2 L$ cross-spectral density matrix expressed as

$$
\mathbf{S}_{\tilde{h}}(\omega)=\left[\begin{array}{ll}
\mathbf{S}^{\Re} \Re(\omega) & \mathbf{S}^{\Re, \Im}(\omega) \\
\mathbf{S}^{\Im, \Re}(\omega) & \mathbf{S}^{\Im, \Im}(\omega)
\end{array}\right],
$$

where the matrix block $\mathbf{S}^{\Re, \Im}(\omega)$ of size $L \times L$ satisfies for all $\omega \in[-\pi, \pi]$

$$
\left[\mathbf{S}^{\Re, \Im}(\omega)\right]_{l p}=\frac{1}{2 \pi} \sum_{u=-\infty}^{+\infty} \mathbb{E}\left\{\Re\left\{\tilde{h}_{l}(k)\right\} \Im\left\{\tilde{h}_{p}(k+u)\right\}\right\} e^{-i u \omega} .
$$

$\Re$ and $\Im$ denotes real and imaginary part, respectively and the matrix blocks $\mathbf{S}^{\Re, \Re}(\omega), \mathbf{S}^{\Im, \Re}(\omega), \mathbf{S}^{\Im, \Im}(\omega)$ are defined analogously to (6). Estimation of this matrix can be performed with common spectral estimation methods (Welch, Burg, Correlogram, etc.) as long as the observation duration of the channel impulse response is much greater than the coherence time of each process $\left\{\tilde{h}_{l}(k)\right\}_{k}$. In most experiments, this duration is on the order of several tens to several hundreds of seconds, which is large in comparison to the coherence time of most UA channels that is usually lower than few hundreds of milliseconds [7]. Note that the common assumption of uncorrelated scattering as well as proper $^{1}$ random processes $\left\{\tilde{h}_{l}(k)\right\}_{k}$ consider $\mathbf{S}_{\tilde{h}}(\omega)$ as diagonal.

Based on the spectral representation (5), it is then possible to draw new realization of the process $\left\{\tilde{h}_{l}(k)\right\}$ using existing techniques for simulating multivariate stationary Gaussian ergodic processes such as those presented in [12]-[14].

As discussed in the introduction, the main drawback of stochastic replay lies in its lack of diversity or flexibility. In the next subsection, we show how it is possible to extend the scope of use of such replay-based modeling strategy by allowing some degree of parameterization. More specifically, it is shown how to distort the second-order statistics of the original TVIR in order to get realizations of a new TVIR that satisfies some desired constraints.

\section{DISTORTING SECOND-ORDER STATISTICS}

\section{A. Theoretical background}

Let $\left\{y_{k}, k \in \mathbb{Z}\right\}$ be a Gaussian random process taking values in $\mathbb{R}^{M}$ and let $p_{\mathbf{Y}_{[-n, n]}}$ denote the joint probability density function of $\mathbf{Y}_{[-n, n]}=\left[y_{-n}, y_{-n+1}, \cdots, y_{n-1}, y_{n}\right]$, the differential entropy rate of $y$ is defined as

$$
\mathrm{h}_{r}(y)=\lim _{n \rightarrow+\infty} \frac{1}{2 n+1} H\left(p_{\mathbf{Y}_{[-n, n]}}\right),
$$

where $H(\cdot)$ denotes the differential entropy [15]. Entropy rates can be considered as a tool for quantitative characterization of dynamic processes evolving in time. For multivariate Gaussian processes, it can be seen as a single metric that carries all the information on the second-order statistics (time fluctuations as well as correlation across individual processes). If $\mathbf{S}_{y}(\omega)$

\footnotetext{
${ }^{1}$ Refer to $[11$, Sec. III-B] for a definition of proper random processes.
} 
denotes the cross-spectral density matrix of $y$, then it can be shown [15] that

$$
\mathrm{h}_{r}(y)=\frac{M}{2} \log (2 \pi e)+\frac{1}{4 \pi} \int_{-\pi}^{\pi} \log \operatorname{det} \mathbf{S}_{y}(\omega) d \omega .
$$

The concept of entropy rate can be extended as a metric for comparing two random processes. Let $\left\{y_{k}, k \in \mathbb{Z}\right\}$ and $\left\{z_{k}, k \in \mathbb{Z}\right\}$ be two random processes, the relative entropy rate between $y$ and $z$ is defined as

$$
D_{r}(y \| z)=\lim _{n \rightarrow+\infty} \frac{1}{2 n+1} D\left(p_{\mathbf{Y}_{[-n, n]}} \| p_{\mathbf{Z}_{[-n, n]}}\right),
$$

where $D(\cdot \| \cdot)$ denotes the relative entropy. If $y$ and $z$ are jointly Gaussian with cross-spectral density matrices $\mathbf{S}_{y}(\omega)$ and $\mathbf{S}_{z}(\omega)$, respectively, then, under some regularity conditions (see [16] for details), their relative entropy satisfies

$$
\begin{aligned}
& D_{r}(y \| z)=\frac{1}{4 \pi} \int_{-\pi}^{\pi}\left(\log \operatorname{det}\left(\mathbf{S}_{y}^{-1}(\omega) \mathbf{S}_{z}(\omega)\right)\right. \\
&\left.+\operatorname{tr}\left[\mathbf{S}_{z}^{-1}(\omega)\left(\mathbf{S}_{y}(\omega)-\mathbf{S}_{z}(\omega)\right)\right]\right) d \omega,
\end{aligned}
$$

where $\operatorname{tr}[\cdot]$ denotes the trace.

\section{B. Relative entropy minimization}

The simulation framework proposed in this work consists in generating realizations of a new multivariate Gaussian random process that satisfies some prescribed constraints, while being "as close as possible" to the reference process $\left\{\tilde{h}_{l}(k)\right\}$. For instance, considering the second-order statistics only, it may be interesting to specify constraints on the Doppler spread in order know to what extent a receiver is able to track the fluctuations of a channel. Similarly, it can be interesting to analyze the impact of the level of correlation between taps on the demodulation performance. Since a realistic prior information is available through the matrix $\mathbf{S}_{\tilde{h}}(\omega)$, it is rather natural to exploit this knowledge to build the new model.

The concept of model proximity can be formally defined through the relative entropy rate between processes, which, as expressed in (10), is also a pseudo-distance between spectral density matrices. Building models with entropy-based criteria is a common procedure in statistical inference [16]-[22]. Such an approach is usually justified on the basis of avoiding the arbitrary introduction of unknown information. ${ }^{2}$ Our modeling strategy can be formalized as follows: let $\left\{y_{k}, k \in \mathbb{Z}\right\}$ be the Gaussian random process taking values in $\mathbb{R}^{2 L}$ that we want to generate, find $\mathbf{S}_{y}^{o}(\omega)$ that solves

$$
\begin{cases}\underset{\mathbf{S}_{y}(\omega)}{\operatorname{minimize}} & D_{r}(y \| \tilde{h}) \\ \text { subject to } & f_{n}\left(\mathbf{S}_{y}(\omega)\right)=\alpha_{n}, n \in 0, \cdots, N,\end{cases}
$$

where $f_{n}$ is some function and $\alpha_{n}$ is either a scalar or a $2 L \times 2 L$ matrix.

To illustrate our modeling approach, we consider for now simple constraints on the channel Doppler spread and the

\footnotetext{
${ }^{2}$ Note that maximum entropy models are not meant to represent the physical reality of the channel but rather a state of knowledge on this reality.
}

channel energy (additional constraints will be considered in an extended version of this paper). More precisely, a particular value $\alpha_{0}$ of average Doppler spread can be specified by constraining the new model $\mathbf{S}_{y}(\omega)$ to satisfy

$$
f_{0}\left(\mathbf{S}_{y}(\omega)\right) \triangleq \int_{-\pi}^{\pi} \omega^{2} \operatorname{tr}\left[\mathbf{S}_{y}(\omega)\right] d \omega=\alpha_{0} .
$$

In addition, the channel energy can also be controlled by setting a new constraint

$$
f_{1}\left(\mathbf{S}_{y}(\omega)\right) \triangleq \int_{-\pi}^{\pi} \operatorname{tr}\left[\mathbf{S}_{y}(\omega)\right] d \omega=\alpha_{1} .
$$

Problem (11) with constraints (12) and (13) can be solved using the method of Lagrange multipliers. According to (10) and since $\frac{-1}{4 \pi} \int_{-\pi}^{\pi} \operatorname{tr}\left[\mathbf{S}_{\tilde{h}}^{-1}(\omega) \mathbf{S}_{\tilde{h}}(\omega)\right] d \omega$ plays no role in the optimization, the Lagrangian is expressed as

$$
\begin{aligned}
& L\left(\mathbf{S}_{y}, \lambda_{0}, \lambda_{1}\right)= \\
& \int\left(\log \frac{\operatorname{det} \mathbf{S}_{\tilde{h}}(\omega)}{\operatorname{det} \mathbf{S}_{y}(\omega)}+\operatorname{tr}\left[\mathbf{S}_{\tilde{h}}^{-1}(\omega) \mathbf{S}_{y}(\omega)\right]\right) d \omega+ \\
& \lambda_{0}\left(\int \omega^{2} \operatorname{tr}\left[\mathbf{S}_{y}(\omega)\right] d \omega-\alpha_{0}\right)+\lambda_{1}\left(\int \operatorname{tr}\left[\mathbf{S}_{y}(\omega)\right] d \omega-\alpha_{1}\right) .
\end{aligned}
$$

It can be shown that the directional derivative of the Lagrangian in direction $\delta \mathbf{S}_{y}$ is given by

$$
\begin{aligned}
\delta L\left(\mathbf{S}_{y}, \lambda_{0}, \lambda_{1} ; \delta \mathbf{S}_{y}\right)=\int & \operatorname{tr}\left[\left(\mathbf{S}_{\tilde{h}}^{-1}(\omega)-\mathbf{S}_{y}^{-1}(\omega)\right.\right. \\
& \left.\left.+\left(\lambda_{0} \omega^{2}+\lambda_{1}\right) \mathbf{I}_{2 L}\right) \delta \mathbf{S}_{y}(\omega)\right] d \omega
\end{aligned}
$$

where $\mathbf{I}_{2 L}$ denotes a $2 L \times 2 L$ identity matrix. Setting this derivative to zero leads to the following solution ${ }^{3}$

$$
\mathbf{S}_{y}^{o}(\omega)=\left[\mathbf{S}_{\tilde{h}}^{-1}(\omega)+\left(\lambda_{0} \omega^{2}+\lambda_{1}\right) \mathbf{I}_{2 L}\right]^{-1} .
$$

The multipliers $\lambda_{0}$ and $\lambda_{1}$ are then obtained by solving the equations (12) and (13). A numerical solution can be found using a gradient-based method such as the one presented in [21].

\section{ILLUSTRATIONS}

Through very basic examples, the purpose of this section is to illustrate the methodology specifically presented in Section III-B. No attempt is made to model realistic channels and application of this method to real UA channels and to performance assessment of UA communication systems is left for future work.

For the sake of simplicity, we consider scenarios where $\left\{\tilde{h}_{l}(k)\right\}$ takes values in $\mathbb{C}^{2}$ (i.e., $L=2$ ) and assume that there is no correlation between the real and imaginary parts of each tap so that $\mathbf{S}_{\tilde{h}}(\omega)$ is block-diagonal. Fluctuations of the taps real and imaginary parts are assumed to be governed by the same spectral density matrix (i.e., $\mathbf{S}^{\Re, \Re}(\omega)=\mathbf{S}^{\Im, \Im}(\omega)$ ). Two different families of Doppler spectrum shape are considered

\footnotetext{
${ }^{3}$ Thanks to the Woodbury identity, the computation of the inverse matrix $\mathbf{S}_{\tilde{h}}^{-1}(\omega)$ can be avoided and (16) can also be written as $\mathbf{S}_{y}^{o}(\omega)=$ $\mathbf{S}_{\tilde{h}}(\omega)\left(\mathbf{I}_{2 L}-\left[\mathbf{I}_{2 L}+\left(\lambda_{0} \omega^{2}+\lambda_{1}\right) \mathbf{S}_{\tilde{h}}(\omega)\right]^{-1}\left(\lambda_{0} \omega^{2}+\lambda_{1}\right) \mathbf{S}_{\tilde{h}}(\omega)\right)$.
} 


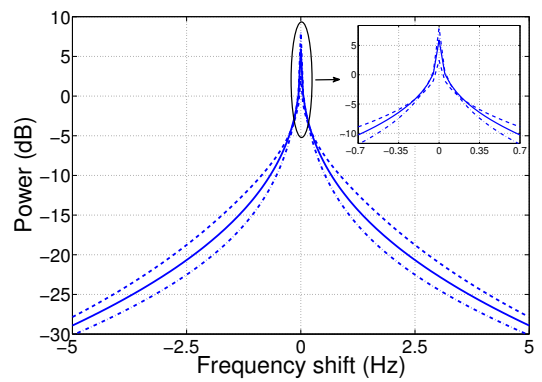

(a)

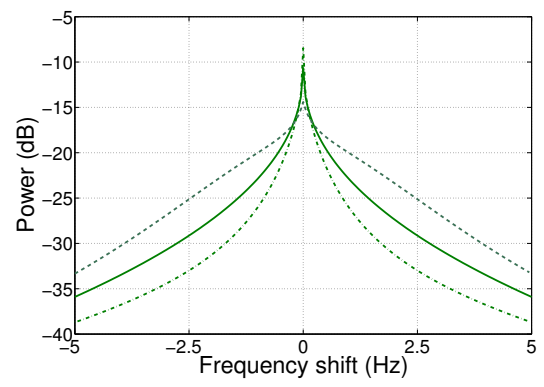

(b)

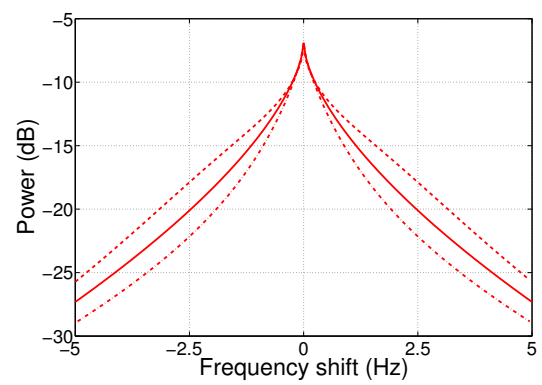

(c)

Fig. 1. Stretched exponential Doppler spectra for a channel with two taps. (a) spectrum of the first tap, (b) cross-spectrum between the two taps, (c) spectrum of the second tap. Plain lines: original spectra, dash-dotted lines: spectra with an average Doppler spread set to $2 / 3$ of the original one, dashed lines: spectra with an average Doppler spread set to $3 / 2$ of the original one.

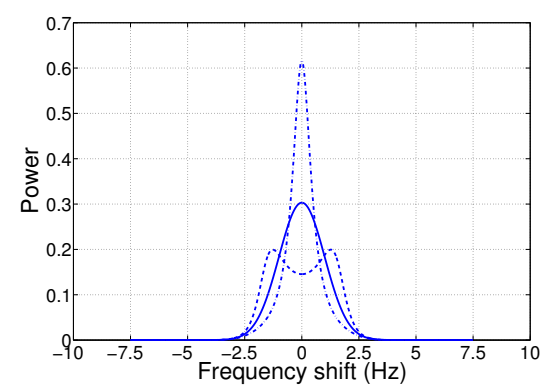

(a)

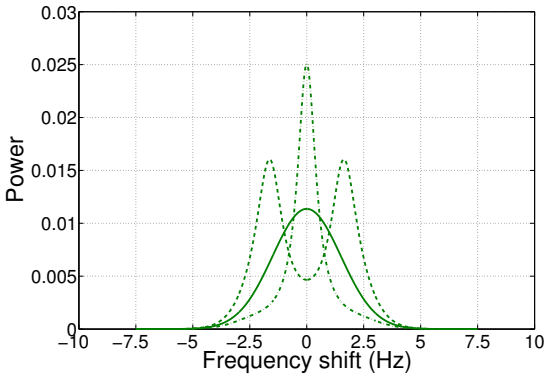

(b)

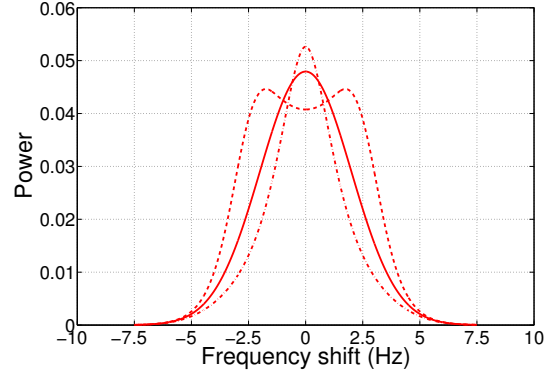

(c)

Fig. 2. Gaussian-shaped Doppler spectra for a channel with two taps. (a) spectrum of the first tap, (b) cross-spectrum between the two taps, (c) spectrum of the second tap. Plain lines: original spectra, dash-dotted lines: spectra with an average Doppler spread set to $2 / 3$ of the original one, dashed lines: spectra with an average Doppler spread set to $3 / 2$ of the original one.

for the illustration and in both cases the two channel taps are correlated (i.e. $\mathbf{S}^{\Re, \Re}(\omega)$ and $\mathbf{S}^{\Im, \Im}(\omega)$ are non-diagonal).

The first example shown in Figure 1 corresponds to the family of sharply peaked Doppler spectra with heavy tails such as those described in [23]. The plain lines of Figure 1-(a), (b) and (c) represents the original spectrum of the first tap (real or imaginary part), the cross-spectrum between the two taps and the original spectrum of the second tap, respectively. The first tap is $5 \mathrm{~dB}$ more powerful than the second one and the correlation level between the two taps is equal to $10 \%$. The dashdotted lines indicate the output of the optimization procedure (11) when the taps fluctuations are artificially slowed down. In this case the average Doppler spread (12) is set to $2 / 3$ of the original one. As for the dashed lines, they correspond to faster taps fluctuations with an average Doppler spread constraint set to $3 / 2$ of the original one. In both cases the average power as expressed by (13) is the same as the original channel. The Doppler spread modification mostly change the peakiness and the tail weight of the spectra stretched exponential shape, the larger the Doppler spread the heavier the tail. It can also be noticed that the second tap becomes slightly more energetic as the Doppler spread increases. This is explained by the fact that the original spread of the second tap is larger than the spread of the first one and as the optimization procedure minimizes the effort to satisfy the specified constraint, if it is told to increase the average spread it will automatically allocate more energy to the second tap.

The second example shown in Figure 2 corresponds to
Gaussian-shaped Doppler spectra as predicted by the theory of underwater sound interacting with the sea surface with a large Rayleigh parameter. The spectra are here plotted in linear scale to better observe the changes. What is specifically interesting to note is the deformation of the spectra that get peaky for small average Doppler spread and that become multimodal as this spread increases. Contrary to the previous example, Gaussian-shaped spectra do not carry much energy in their tails, it is therefore less demanding in term of entropy change to modify the original Doppler spectra around their mode rather than around their tails.

\section{Conclusions And Perspectives}

UAC channel modeling as defined in the proposed parametric and replay-based framework may be a way to find a good compromise between model realism, parameterization simplicity and flexibility. Thanks to the formalism of relative entropy minimization between random processes, we can control some statistical properties of the simulated channel while being close to realistic TVIR. The work presented in this paper sets the general concept but additional research has to be conducted to assess the applicability of the proposed framework to real data and to further extend its possibility. For instance, constraints on the correlation level between channel taps may be interesting to add in order to measure their impact on modems performance and, more largely, generalized moments constraints, as formalized in [16], [22], [24], [25], could also be considered. 


\section{REFERENCES}

[1] P. van Walree, P. Jenserud, and M. Smedsrud, "A Discrete-Time Channel Simulator Driven by Measured Scattering Functions," IEEE J. Sel. Areas Commun., vol. 26, no. 9, pp. 1628-1637, 2008.

[2] F.-X. Socheleau, C. Laot, and J.-M. Passerieux, "Stochastic Replay of non-WSSUS Underwater Acoustic Communication Channels Recorded at Sea," IEEE Trans. Signal Process., vol. 59, no. 10, pp. 4838-4849, 2011.

[3] R. Otnes, P. van Walree, and T. Jenserud, "Validation of Replay-Based Underwater Acoustic Communication Channel Simulation," IEEE J. Ocean. Eng., vol. 38, no. 4, pp. 689-700, 2013.

[4] J.-M. Passerieux, F.-X. Socheleau, and C. Laot, "On the capacity of the Underwater Acoustic Communication Channel under Realistic Assumptions,", in Proc. IEEE European Wireless, Apr. 2011, pp. 1-6.

[5] F.-X. Socheleau, M. Stojanovic, C. Laot, and J.-M. Passerieux, "Information-Theoretic Analysis of Underwater Acoustic OFDM Systems in Highly Dispersive Channels," Journal of Electrical and Computer Engineering, 2012, Article ID 716720.

[6] J.-M. Passerieux, F.-X. Socheleau, and C. Laot, "Achievable rates over doubly selective rician-fading channels under peak-power constraint," Wireless Communications, IEEE Transactions on, vol. 12, no. 2, pp. 586-594, February 2013.

[7] P. van Walree, "Channel sounding for acoustic communications: techniques and shallow-water examples," Research report, Norwegian Defence Research Establishment (FFI), 2011.

[8] F.-X. Socheleau, J.-M. Passerieux, and C. Laot, "Acoustic Modems Performance Assessment via Stochastic Replay of at Sea Recorded underwater Acoustic Communication Channels," in Proc. Underwater Acoustic Measurement: Technologies and Results, Jun. 2011.

[9] R. Otnes, P.A. van Walree, and T. Jenserud, "Erratum to validation of replay-based underwater acoustic communication channel simulation," IEEE J. Ocean. Eng., vol. 38, no. 4, pp. 809-809, Oct 2013.

[10] F.-X. Socheleau, J.-M. Passerieux, and C. Laot, "Characterisation of Time-Varying Underwater Acoustic Communication Channel with Application to Channel Capacity," in Proc. Underwater Acoustic Measurement, Jun. 2009.

[11] F. D. Neeser and J. L. Massey, "Proper Complex Random Processes with Applications to Information Theory ," IEEE Trans. Inf. Theory, vol. 39, no. 4, pp. 1293-1302, 1993.
[12] G. Deodatis, "Simulation of Ergodic Multivariate Stochastic Processes," Journal of Engineering Mechanics, vol. 122, no. 8, pp. 778-787, 1996.

[13] L. Chen and C. W. Letchford, "Simulation of Multivariate Stationary Gaussian Stochastic Processes: Hybrid Spectral Representation and Proper Orthogonal Decomposition Approach," Journal of Engineering Mechanics, vol. 131, no. 8, pp. 801-808, 2005.

[14] Q. Ding, L. Zhu, and H. Xiang, "An efficient ergodic simulation of multivariate stochastic processes with spectral representation," Probabilistic Engineering Mechanics, vol. 26, no. 2, pp. 350 - 356, 2011.

[15] T. Cover and J. Thomas, Elements of Information Theory, Wiley, 1991.

[16] A. Ferrante, C. Masiero, and M. Pavon, "Time and spectral domain relative entropy: A new approach to multivariate spectral estimation," Automatic Control, IEEE Transactions on, vol. 57, no. 10, pp. 25612575, Oct 2012

[17] E.T. Jaynes, "Information Theory and Statistical Mechanics," Physical review, vol. 106, no. 4, pp. 620-630, 1957.

[18] J. Shore and R. Johnson, "Axiomatic Derivation of the Principle of Maximum Entropy and the Principle of Minimum Cross-Entropy," IEEE Trans. Inf. Theory, vol. 26, no. 1, pp. 26-37, 1980.

[19] J. P. Burg, Maximum Entropy Spectral Analysis, Ph.D. thesis, Department of Geophysics, Stanford University, 1975.

[20] M. Debbah and R. R. Muller, "MIMO Channel Modeling and the Principle of Maximum Entropy," IEEE Trans. Inf. Theory, vol. 51, no. 5, pp. 1667-1690, 2005.

[21] F.-X. Socheleau, C. Laot, and J.-M. Passerieux, "Concise Derivation of Scattering Function from Channel Entropy Maximization," IEEE Trans. Commun, vol. 58, no. 11, Nov. 2010.

[22] A. Ferrante, M. Pavon, and M. Zorzi, "A maximum entropy enhancement for a family of high-resolution spectral estimators," Automatic Control, IEEE Transactions on, vol. 57, no. 2, pp. 318-329, Feb 2012.

[23] P. A. van Walree, T. Jenserud, and R. Otnes, "Stretched-exponential doppler spectra in underwater acoustic communication channels," The Journal of the Acoustical Society of America, vol. 128, no. 5, 2010.

[24] T.T. Georgiou, "Relative entropy and the multivariable multidimensional moment problem," Information Theory, IEEE Transactions on, vol. 52, no. 3, pp. 1052-1066, March 2006.

[25] A Ferrante, M. Pavon, and F. Ramponi, "Hellinger versus kullbackleibler multivariable spectrum approximation," Automatic Control, IEEE Transactions on, vol. 53, no. 4, pp. 954-967, May 2008. 